Euskal ikerketen aldizkaria | Revue d'études basques |

Revista de estudios vascos | Basque studies review

$20 \mid 2017$

Numéro $X X$

\title{
Autour des conditionnels passés analytiques du basque
}

\section{Georges Rebuschi}

\section{OpenEdition}

\section{Journals}

Édition électronique

URL : https://journals.openedition.org/lapurdum/3569

DOI : 10.4000/lapurdum.3569

ISSN : 1965-0655

\section{Éditeur}

IKER

Édition imprimée

Date de publication : 1 janvier 2017

Pagination : 239-257

ISBN : 978-2-95534-135-3

ISSN : $1273-3830$

Référence électronique

Georges Rebuschi, «Autour des conditionnels passés analytiques du basque », Lapurdum [En ligne] 20 | 2017, mis en ligne le 01 janvier 2021, consulté le 03 septembre 2021. URL : http://

journals.openedition.org/lapurdum/3569; DOI : https://doi.org/10.4000/lapurdum.3569 


\section{Autour des conditionnels passés analytiques du basque}

Georges REBUSCHI

Sorbonne-Nouvelle \& LACITO

Ur-garbizura, iturburura

(Oihénart, proverbe 457)

\section{Introduction ${ }^{1}$}

\subsection{But de ce travail}

Les formes qui expriment le conditionnel passé n'ont, à ma connaissance, jamais été étudiées en ou pour elles-mêmes. Je tente donc d'en établir la complexité à travers les dialectes et les époques (sans être sûr, malheureusement, du caractère exhaustif des données). Au lieu de «piocher» au hasard dans des textes variés, j'ai utilisé les nombreuses traductions bibliques (parfois restreintes au seul NT, voire à un seul Evangile) dont on dispose depuis quatre siècles et demi, car elles se paraphrasent nécessairement plus ou moins - le tout, évidemment, sans chercher à soulever de quelconques discussions de théologie, ni même de traductologie.

La section 2 donnera quelques indications sur la fréquence relative des trois types de formes les plus fréquentes (combinaisons d'un participe, perfectif ou prospectif, et d'un auxiliaire au passé, en $z$-, et porteur ou non du préfixe -ke). La section 3 présentera trois autres combinaisons, dans lesquelles l'auxiliaire porte par contre un préfixe -l, généralement considéré comme ne relevant que du conditionnel présent-futur. Plusieurs traductions d'un même passage de la Genèse montreront au § 4 les rapports entre ces six types et l'interprétation non conditionnelle, normale également, de la plupart de ces combinaisons. En 5, la valeur

1. C'est en étudiant les textes mis à la disposition du public par l'énorme travail fourni par Xarles V. et ses collègues (Aurrekoetxea et al. (2004, 2007) que l'idée m'est venue d'utiliser systématiquement les traductions religieuses si nombreuses dont on dispose en langue basque. Je suis donc particulièrement heureux de lui offrir cette trop courte étude. Par ailleurs, je remercie vivement Marie Pourquié pour ses remarques très constructives sur une version antérieure de ce travail. 
fréquentative de la combinaison prospectif + aux. au passé sans -ke, sera très brièvement exposée. Enfin, les auxiliaires fléchis en -l étant particulièrement typiques du souletin et du biscayen, l'attention du lecteur sera attirée sur d'autres phénomènes qui suggèrent (peut-être) une connexion ancienne, les données périphériques pouvant souvent, dans de très nombreuses langues, être considérées comme moins innovatrices que les autres.

\subsection{Généralités sur la conjugaison basque}

Pour les verbes qui peuvent se conjuguer directement, et quand le ou les arguments (absolutif et, le cas échéant, ergatif) est/sont de 3e personne (je laisse de côté le datif, car il n'est jamais représenté par un préfixe), les formes verbales fléchies (FVF) disposent de trois préfixes qui s'opposent, (la), et de deux suffixes, (1b) qui peuvent par contre tant se combiner entre eux qu'avec les formes construites avec les affixes de (la).

(1) a d-/z-/l-

b $-k e,-(e / a) n$

Traditionnellement, on associe $d$ - au présent, / z-au passé, et / l-à l'éventuel, ce qui, mettant deux notions temporelles sur le même plan qu'une notion modale, n'est pas sans difficulté. D’autre part, -ke relève du potentiel et/ou de la prédiction plus ou moins forte (donc de l'ultériorité dans le domaine temporel, et de la probabilité dans le domaine modal), tandis que -(e/a)n est prioritairement une marque de passé qui est absorbée soit par un suffixe phonologiquement identique, mais lié à l'environnement syntaxique (questions indirectes, relatives, certaines complétives), soit par le suffixe complétif - (e/a)la.

Cela dit, lorsque l'argument ergatif est de lère ou 2e personne et que l'argument absolutif reste (comme ci-dessus) de 3e personne, au passé et à l'éventuel, les préfixes $z$ - et l- mentionnés en (la) supra sont remplacés par des préfixes personnels qui relèvent de la même série, par ex. 1 sg. : nin(d)-/ nen-; 2 sg. : hin(d)- / hen-. Cette caractéristique permet de regrouper partiellement les FVF en $z$ - et $l$ - comme renvoyant à un prédicat dont la dénotation (état, action, événement) ne relève pas du présent actuel. ${ }^{2}$

Noter que si la combinaison de ces affixes ne donne pas toujours des FVF bien formées, la liste exhaustive des formes jugées standard, par ex. avec le verbe izan ${ }_{2}$ ukan 'avoir': du 'il l'a', zuen 'il l'avait, luke 'il l'aurait [cond.], zukeen 'il l'aurait (eu) [cond. passé], ou radicalement impossibles ( $\left.{ }^{*} z u,{ }^{*} z u k e,{ }^{*} l u\right)$ ne peut pas être établie, en raison de l'existence de formes marginales, dialectalement limitées, et/ou archaïques. Ainsi que faire de luen 'qu'il eût', subjonctif passé attesté par exemple chez Liçarrague (1571) ? Et que faire du préfixe ba- 'si' hypothétique, puisqu'il est non seulement compatible avec les formes du et zuen mentionnées

2. En conséquence, quand on a affaire à une variété de basque dont certaines propriétés morphologiques ne sont pas identifiables (le tout dépendant de ce qu'offrent les textes qui ont survécu), et que d'autre part un suffixe comme -(e/a)n apparaît (qui peut, on l'a dit marquer aussi la subordination ou l'exclamation par ex.), il n'est pas toujours possible de savoir si la FVC aurait, pour des arguments de $3^{e}$ personne uniquement, un préfixe l- ou $z$-. Je me concentrerai donc sur des ex. à cette 3 e personne. 
à l'instant, mais qu'il rend *lu acceptable et même normal: balu 's'il l'avait' (hypothétique du présent-futur)?

Passons à la conjugaison analytique ordinaire (souvent appelée «périphrastique » ${ }^{3}$ ), qui nous concerne au premier chef. Laissant de côté les tours mentionnés dans l'alinéa précédent, on peut combiner a priori n'importe quelle forme fléchie des auxilaires izan , être' et izan / ukan 'avoir' avec l'un des trois participes marqués aspectuellement par un suffixe: le perfectif (-tu), l'imperfectif (-tzen) et le prospectif (-tuko). Or ces terminaisons ont toutes des variantes, mais ce qui compte ici, c'est qu'on se trouve face aux mêmes difficultés qu'en ce qui concerne les combinaisons des affixes de (la) et (b) mentionnées supra. En effet, en biscayen, (i) tant le perfectif (en -ten maintenant) que le prospectif se suffixent au p. perfectif, et (ii) ce dialecte n'emploie généralement pas le radical avec les auxiliaires défectifs, mais la forme 'perfective' en -tu; ce dialecte a donc neutralisé totalement l'opposition entre part. perfectif et radical; quant au guipuzcoan non-«batuisé», il occupe une position intermédiaire entre les dialectes du Nord et l'euskara batua, conservant la neutralisation du radical verbal et du part. perf. au profit de ce dernier, mais dérivant le part. imperf. du radical comme le fait le basque standard. ${ }^{4}$

Il faut mentionner pour mémoire deux auxiliaires défectifs, *edin (intransitif) et *ezan (transitif), justement défectifs parce qu'ils n'ont pas de formes non-fléchies (cf. les aux. modaux de l'anglais will, may etc.). Je n'en parlerai plus (sauf en 6.2), car ils ne servent usuellement qu'à décrire des états ou événements non-actuels, mais visés ou envisagés comme possibles à partir du moment d'énonciation ou d'un moment antérieur (comme dans les subjonctifs ou potentiels passés), et qu'ils sont associés non aux participes aspectuels (voir ci-dessous), mais aux radicaux verbaux, ne pouvant «normalement» pas servir à construire des conditionnels passés, irréels - mais peuvent par contre contribuer à la formation de FVF potentielles, hypothétiques (ou formes conditionnantes des protases) et des conditionnels proprement dits (i.e. les formes sémantiquement conditionnées des apodoses) du présentfutur. Trois exemples: (i) ikus dezake / zezakeen 'il peut/pouvait le voir'; (ii) ikus baleza, har

3. Souvent appelée "périphrastique», mais je suis ici les suggestions de Hualde et al. (2003): je conserve ce terme pour les combinaisons dans lesquelles le verbe lexical associé à la FVF n'est ni un participe nu (associé aux FVF décrites ci-dessus) ni un radical (associé aux aux. défectifs), mais soit un participe perfectif suffixé (d'une marque de nombre, du morphème partitif, ou de -(e)ta 'après avoir + p.pé' soit un mot comme nahi 'vouloir/volonté', ou behar 'devoir/besoin', dont la syntaxe est assez particulière, d'où il suit que, dans ces cas, une FVF comme du ou da est un verbe plein, et non un auxiliaire.

4. Illustrations rapides. Pour (i), billatuten ebenak vs. billatzen zutenak 'ceux qui le cherchaient' (Uriarte, resp. sa trad. bisc. de Mt 2,20, et l'une de ses deux trad. guip. du même passage); pour (ii), Mt 10,28 par le même traducteur: (bildur izan zakijuez) arimia ta gorputza galdu leikezanari... (bisc.) '(craignez) celui qui peut perdre l'âme et le corps'(sur le pot. en l-, voir plus loin). La traduction standardisée et celle en navarro-labourdin d'Ezkila' (1974) ont par contre pertsona osoa infernuan honda Ø dezakeenari et arima eta gorputza gehenean gal $\emptyset$ dezakeenaren beldur izan zaitezte resp., tandis qu'en guipuzcoan, Uriarte traduisait comme attendu: anima eta gorputza... galdu ditzakeanaren beldur izan zaitezte. 
lezake 's'il le voyait, il le prendrait / pourrait le prendre'; (iii) (nahi dute) har dezan 'ils veulent qu'il/elle le prenne'.

\section{Les conditionnels passés à préfixe -z sur l'auxiliaire}

\subsection{Généralités}

Il en existe trois types, bien recensés dans Euskaltzaindia (2005) par exemple. Ils sont rappelés dans le tableau 1 ci-desous. Leur seul intérêt ici est d'en relativiser la distribution et la fréquence dans l'espace et le temps.

\section{Tableau 1}

Formes verbales conjuguées illustrées par l'équivalent basque usuel de 'il l'aurait pris' dans l'apodose d'une phrase complexe dont la protase pourait être: Ikusi (izan/ukan) balu... 'S'il l'avait vu...'

TYPE 1: hartu zukeen part. perf. + aux passé avec addition de -ke

TYPE 2: hartuko zuen, part. prospectif + aux. passé sans plus

TYPE 3: hartuko zukeen part. prospectif + aux. passé avec -ke

\subsection{Les traductions religieuses, du $16 \mathrm{e}$ au 21 e siècles}

Dans la tradition cispyrénéenne, deux tours couvrent l'essentiel du champ sémantique considéré, à savoir, le TYPE 1 et le TYPE 2. Il faut cependant ajouter le TYPE 3, car ces formes, quoique plus rares, sont apparues bien avant la normativisation venue du Pays basque péninsulaire.

Dans le NT de Liçarrague (1571), seul le type 1 est utilisé ${ }^{6}$ (sauf lorsqu'un «cond. passé » français est en réalité un futur translaté dans le passé ${ }^{7}$ ). Mais c'est surtout en souletin qu'il a été préservé: ukhan zukeen, dans la grammaire labourdine d'Ithurry (1895: p. 381) est

5. La duplication de l'aux. transitif au perfectif (ukan au Nord, izan au sud) ne fait que souligner l'antériorité de l'événement ou l'état fictif ou irréel auquel la protase fait allusion.

6. A une exception près (sauf omission involontaire), Mt 23,30: Baldin gure aitén egunetan içan baguina, ezquinén hayén lagun içanen Prophetén odolean 'Si nous avions été au temps de nos pères, nous ne nous serions pas joints avec eux pour répandre [lit. : nous n'aurions pas été leurs compagnons dans] le sang des prophètes.'

7. Par ex. Mk 3,2: gogoatzen çutén eya Sabbathoan sendaturen çuenez 'ils l'observaient (pour voir) s'il le guérirait (le jour du) Sabbat.' 
simplement traduit par 'il l'avait probablement eu'. ${ }^{8},{ }^{9}$ Le type 2 , lui, apparaît dès la fin du $17^{e}$ siècle. Quant au type 3, c'est surtout dans le NT labourdin de Haraneder qu'il se manifeste dans les écrits relativement anciens; curieusement, c'est, par contre, uniquement en basnavarrais que ce type est utilisé dans les deux études dialectales présentées en 2.3.

Pour prendre un cas particulièrement clair, dans les vingt traductions de Jean 11, 21 que j'ai trouvées, la plus ancienne, de Liçarrague, et elle seule, donne le type 1, et dix-huit sont du type 2 (dont onze relèvent de la zone cis-pyrénéenne, une du haut-navarrais méridional, six du basque «central» ou du basque récemment « unifié», et une du biscayen du début du 19 e s.; pour la dernière, qui est de type 6, cf. 3.3. infra).

L'équivalence sémantique entre les types 1 et 2, contestée par certains puristes, est soulignée par le fait qu'ils se retrouvent, inversés, dans les deux versets suivants des Psaumes (54/55,12-13), d'une part chez Duvoisin en labourdin, et d'autre part chez Archu en souletin (la fin du participe et l'éventuel -ke sont en gras):

(1) Duvoisin: 13. Ezen baldin ene etsaiak madarikatu izan banindu, jasan nukeen segurki [T.1]; eta gaitzirizkotan nindaukana, ene kontra goraki mintzatu izan balitz, gordeko nintzen [T.2] eiki haren ganik. Archu: 12. Ene etsaiak gaitzetsitü banündü, enizün hitzik ere erranen.[T.2]. 13. Eta hügüntzen nündienak nitzaz gaizki handi erran balü, behar bada gorde nündükezün harenganik. [T.1] 'Si mon ennemi m'avait maudit/outragé, je l'aurais supporté/n'aurais rien dit; et si celui qui me hait avait proféré contre moi des insolences/menaces, je me serais gardé de lui.'

Autre exemple, les deux cond. passés de Mat. 11,21+23: sur 30 traductions (et non plus 20 ! ], il y a trois occurrences du type 1, trente-six du type 2 , et deux seulement du type 3 (il y a de plus trois constructions atypiques, cf. la section 3, toutes les autres présentant toutes

8. Ce type apparaît en biscayen dans la toute première description du basque péninsulaire, due à Micoleta (ms. 1653, éd. de 1897, p.9) : a isan satekea 'aquél huviera sido', avec, indépendamment, absence du - $n$ du passé [c'est une question qui se pose pour de nombreux parlers et mériterait un travail à part - cf. chez le même Micoleta: ni nintsá 'yo era' (1897: 7), en face du type 2 employé avec un verbe transitif: ak egingo Øebea 'aquél huviera hecho' (id.) - le symbole $\varnothing$ représente ici l'allomorphe biscayen usuel de $z$ - avec les FVF transitives.

9. Pour le labourdin d'un siècle et demi plus tôt, Harriet (1741: 190) opposait par l'étiquetage le "parfait conjonctif», hark thebekhatu zukeien 'il aurait défendu' [au sens d'interdire'] au «plus-que-parfait conjonctif» hark thebekhatuko zuen 'il eût défendu', ce qui indique que les deux formes étaient encore en usage, mais rien n'indique de différence sémantique. Cent ans plus tard, chez Hiriart (1840:87-88), si on laisse l'opposition purement aspectuelle de côté (la marque d'accord -a sur le part. perfectif oriente vers l'état résultant de l'action), on trouve uniquement: "kantatua ukhanen zuen 'il aurait chanté' [mais] on dit aussi: kantatuko zuen 'il eût chanté’». En d'autres termes, le type 1 avait déjà pratiquement disparu en (navarro-?) labourdin... 
des verbes forts, conjugués synthétiquement). On peut illustrer une partie de ces données avec Duvoisin encore pour ce passage de Mt. :

(2) [v. 21]... zeren Tirren eta Sidonen gerthatu izan balire zuen erdian egin diren sendagailak, aspaldi huntan ... egin baitzituzketen [1] urrikizko eginkariak. 'Si les guérisons miraculeuses qui ont été faites à Tyr et Sidon s'étaient produites parmi vous, il y a longtemps qu'elles [ce villes] auraient fait pénitence.' [v. 23] ... zeren Sodomarren artean gerthatu izan balire hire erdian egin diren sendagailak, egungo eguneraino iraunen baitzuten [2] menturaz. '... parce que si les miracles qui ont été faits en ton sein s'étaient produits à Sodome, peut-être aurait-elle perduré jusqu'à aujourd'hui.'

\subsection{Résultats de deux enquêtes au Pays-Basque Nord}

\subsubsection{Le « Recueil Bourciez» (1895-96)}

Ce sont les mêmes données que celles trouvées ci-dessus que fournit le Recueil Bourciez (Aurrekoetxea et Videgain, 2004), qui contient environ 145 traductions exploitables d'une version laïcisée de la parabole de l'enfant prodigue, donnée à traduire dans les années 1890 aux instituteurs de la plupart des communes basques dans leur variété locale ${ }^{10}$. Une seule phrase à traduire contenait un cond. passé: 'Il avait quelquefois si grand faim qu'il aurait bien mangé ces feuilles de choux [...].' Les uniques combinaisons d'un participe et d'un aux. présentes dans cet ouvrage sont les trois non-marquées décrites supra. Leur proportion est donnée dans le tableau 2 ci-après:

\section{Tableau $2^{11}$}

TYPE 1 , jan zukeen/ zituzkeen (selon que l'objet direct est au sg. ou au pl.):

Labourd, 3/41; Basse-Navarre, 6/72; Soule, 18/34; pourcentage total: 18,3\% des traductions.

TYPe 2, janen zuen / zituen, Lab., 35/41; B-N, 60/72; Soule, 14/34; pourcentage total: $73,5 \%$.

10. Dans ce recueil, il est difficile de cerner si le traducteur possédait bien le parler de l'endroit où il était en poste. Qui plus est, il faudrait refaire ces décomptes en tenant compte du statut dialectal des communes: on sait que les parlers bas-navarrais orientaux étaient usités, au moins jusqu'à la fin du $19^{e}$ siècle, dans tout le nord de la Basse-Navarre, et même dans le Labourd, sur les rives de l'Adour.

11. Dans ce tableau et dans le suivant, le total des pourcentages n'atteint pas $100 \%$ car certaines des traductions ne représentent pas de conditionnel passe. 
TYPe 3, janen zukeen/zituzkeen.

Lab., 1; B-N, 6; S., $\varnothing$;

pourcentage total : $4,7 \%$.

On remarquera une certaine asymétrie: le type 1, le plus ancien, n'était déjà vraiment plus vivant qu'en Soule, où il représentait malgré tout un peu plus de la moitié des versions ${ }^{12}$; inversement, il ne représentait que 9\% des zones centrales et occidentales. Par contre, dans ces deux dernières régions, le type 2 atteignait près de $85 \% .^{13}$

\subsubsection{L'enquête NORANTZ (2009-2011)}

L'enquête sociolinguistique récente «Norantz », effectuée au Pays basque continental par l'équipe IKER du CNRS, donne des résultats comparativement intéressants: en regardant dans le détail la traduction (proposée à cinquante-neuf locuteurs) des quatre phrases françaises qui contenaient un cond. passé avec argument(s) non-datif(s) à la $3^{e}$ personne, en tenant compte du lieu de résidence des informateurs, et en éliminant les quelques traductions qui ne relèvent pas de notre problématique (l'emploi erroné d'un des conditionnels du présent-futur, ou encore verbes forts, fléchis synthétiquement), on obtient les résultats suivants pour les quatre traductions prises en bloc:

\section{Tableau 3}

TyPE 1, ikusi zuke(e)n 'il [l'] aurait vu(e)' (et trois autres phrases semblables): Labourd, $\varnothing / 48$; Basse-Navarre, $\varnothing / 92$; Soule, $3 / 35$; locuteurs/trices de dialecte nonspécifié ou mêlé: $\varnothing / 42$; pourcentage total : $1,4 \%$

TYPe 2, ikusiko zuen

L., 47/48; B-N, 83/92; S., 31/35; dial. mêlé etc.: 40/42; pourcentage total: 92,6\%

12. Zavala (1848:22) atteste de cette formation à l'opposé géographique du souletin, i.e. en biscayen (sur $\varnothing$, cf. la note 8) mais il en souligne le caractère hautement conjectural dans son dialecte, traduisant itxi Øeukean sakramentu bakar bat par: 'pudiera haber dejado un solo sacramento'.

13. Pour le passage correspondant dans le NT proprement dit, seules les traductions continentales ont un conditionnel passé associé à nahi 'vouloir/volonté'. Sans entrer dans les détails, car la forme conjuguée relève de la conjugaison forte ou synthétique, on trouve: nahi zuk(e)en/zükian au $18^{\mathrm{e}} \mathrm{s}$. (Haraneder) et dans trois trad. récentes (Casenave 1987 et 2002, Etchehandy 2008), et nahiko zuen aux $19^{e}$ et $20^{e}$ siècles (Harriet, Léon, 'Ezkila'): il n'y a ici aucun ex. de nahiko zukeen; mais Léon (1946) l'emploie bien dans sa trad. de Jn 7,44: Batzuek nahiko zuketen ere preso hartu, 'certains auraient voulu le mettre en prison'. 
TYPE 3, ikusiko zuke(e)n

L., $\varnothing / 48$; B-N, 4/92; S, $\varnothing / 35$; dial. mêlé etc.:2/42; $\quad$ pourcentage total : 2,8\%

La réduction de l'emploi du type 1 et, corrélativement, l'élargissement de celui du type 2 , sont particulièrement remarquables quand on prend en compte l'âge des informateurs et informatrices: les quatorze personnes âgées de 70 ans et plus (qui n'ont évidemment pas été en ikastola), ont traduit les quatre phrases en utilisant le type 2 à 100\% des formes attendues, les seules traductions non prises en compte étant trois verbes conjugués synthétiquement, et un seul conditionnel présent/futur au lieu du passé. Cette réduction de la fréquence du type 1 semble avoir conduit, en navarro-labourdin au moins, à une réinterprétation de la combinaison p.passé + aux+ke comme marquant simplement une supputation, l'expression d'une conjecture de la part du locuteur. C'est en tout cas ce que l'on peut inférer déjà de Lafitte (1944): voir par ex. sa traduction de galdu zateken et galdu zuken dans le tableau des conjugaisons entre les pp. 338 et 341: 'il devait s'être perdu' (intransitif), 'il a dû (le) perdre' (transitif). ${ }^{14}$

\subsection{Remarques complémentaires sur le type 3}

Ce type, quoique plus typique des écrits péninsulaires, est aussi mentionné comme «temps 30, Parfait propositif: ikusiren zukeen » [et même] 31 parfait propositif composé: ikusi ukanen zukeen 'il l'aurait vu'» par Gavel \& Lacombe (1937).

Noter que chez Larramendi (1729), jangó zéuen/zuen était simplement un conditionnel présent-futur: '[él] comería' (p. 81) alors qu'avec la duplication de l'aux., jan izangó zéuen/ zuen, on obtenait bien un conditionnel passé '[él] lo habría comido'. Pour le biscayen, voir par ex. Azkue (1896): p. 62 ibili izango zan 'había de haber o habría de haber andado'; p. 124 Zeinbat mutil egon izango dira 'cuántos habrán estado'; et aussi : p 134 bildur izango litzake 'habría de temer o temería'; dans cet ouvrage, rien d'explicite n'est dit sur le conditionnel passé, mais l'avant-dernier exercice du tout dernier chapitre (pp. 291-2) propose malgré tout à la traduction, la phrase suivante, dont l'interprétation est évidente: Iakin baneu zer zan, eneban erosiko ['si j'avais su ce que c'était, je ne l'aurais pas acheté'].

L'extension du type 3 Outre-Bidassoa doit sans doute beaucoup à Altube (1929: §191) qui condamne violemment le type 2 si fréquent partout, sauf peut-être en biscayen - et encore: l'année précédente, Zamarripa (1928: 95) traduisait artuko eban par 'él) hubiera tomado' mais pouvait aussi dupliquer líauxiliaire (le premier, non-marqué, étant au perfectif et le second

14. Cependant, notre type 2, hartuko nuen 'je l'aurais pris' est quand même compté comme l'une des [deux] formes «les plus usitées» (Laf .1944/1960: p. 381, § 720). Voir aussi cette phrase de Lafitte écrivain (1990 - texte écrit entre 1934 et 1967), qui confirme cette interprétation: Berrondon holako zerbait pasatu zateken duela ehun bat urte, ezen bertsu hau gelditu da... 'Quelque chose de semblable avait dû se passer à Berrondo il y a une centaine d'années, car cette strophe est restée:....' 
étant au prospectif: eldu izango zan '(él) habría llegado').

\subsection{Transition: introduction aux formes du passé en l-}

Par ailleurs, dans le corpus Norantz, les autres formes analytiques relevées comportent un l- initial plutôt que le $z$ - du passé qui est apparu partout jusqu'ici. Concrètement, un informateur labourdin a traduit 'Cela aurait paru bizarre à Beñat' par: Beñati bitxi egingo Leizaioken (lit. 'ça lui aurait fait bizarre'), où le part. prospectif est associé à un aux. abs.-datif suffixé de -ke puis de -(e)n, comme dans le type 3, mais où l'initiale est un l-, généralement associé au conditionnel présent-futur, du moins dans la plupart des parlers navarro-labourdins et «centraux»: c'est le type 6 décrit infra. L'autre forme atypique est donnée par une locutrice de B-N, pour la même phrase: bitxia iduriko sitzaion Beñati, où manque le morphème -ke. C'est vers ces conditionnels passés en l-, passés généralement sous silence (par ex. dans Hualde et al. 2003, ou Euskaltzaindia 2005) que nous allons nous tourner maintenant - laissant toutefois cette toute dernière forme de côté, car je ne l'ai trouvée nulle part ailleurs.

\section{Les conditionnels passés à préfixe $-l$ sur l'auxiliaire}

\subsection{Le type 4: hartu luke}

Ce type, mis en avant par Lafitte (1944) (peut-être poussé par la confusion de vocabulaire en grammaire française, les participes perfectifs y étant traditionnellement appelés "passés») passe souvent pour une illusion. ${ }^{15}$ Mais ce n'est pas tout à fait le cas, comme le montrent (3), en labourdin classique, et (4), en navarro-labourdin plus récent, qui reprend le même thème:

(3) Gerla hunek, aphur bat ere gibelatzen gaitu bekhatu egitetik, bertzela egin Luke gureak. 'Cette guerre [que nous fait notre conscience] nous retient un peu de commettre le péché, autrement il en serait fait de nous.' (Axular 1643, \$274 de la rééd. de 1964; trad. de Villasante: 'de lo contrario, estábamos perdidos').

(4) Laphitz (1876) - Ah! zangorik ez hartan sar! Hartan sarthuz zureak egin ㄴuke. 'N'y mettez pas vos [lit. 'de'] pieds! En les y mettant, il en serait fini de vous.'

15. Comme on le verra, les deux autres types en l-portent une marque de passé, le suffixe -en. Ce qui est étonnant avec ce type 4, c'est l'absence de toute marque explicitement temporelle. Or si l'on considère le Type 2, on voit que si, d'une part, c'est la protase (en l- elle aussi) qui force une interprétation conditionnelle de l'apodose (sinon, elle représente un futur translaté dans le passé, voire autre chose (cf. les sections 4 et 5), c'est, d'autre part, et dans l'autre sens, l'apodose qui donne vraiment la localisation temporelle à la phrase entière. Tout se passe donc ici comme si l'aspect perfectif associé à un auxiliaire en -l (suffixe dont on verra plus tard les rapports avec le passé), suffisait pour remplir cette fonction. 
Du côté des grammaires, cette combinaison n'était pas passée inaperçue. Ainsi, Inchauspé (1853) notait-il l'existence des types 4 et 1 respectivement, en traduisant à la fois eskéntü Lüke (p. 294 et ailleurs) et eskéntü züKían (type 1) par 'il aurait offert' (p. 4), ou encore p. 18 gáldü Lizáte ${ }^{16}$ par 'il se serait perdu'; il propose de plus (p. 18) une nuance temporelle qui peut sembler évidente: galdü LِüKE 'il aurait perdu (actuellement)', T4, vs. gáldü züKían 'il aurait perdu (autrefois)', T1, mais ne correspond pas vraiment aux exemples relevés dans divers textes, cf. (1) supra). Toujours en souletin, Archu (1853: 42), la cite dans la version de 1868 (p. 38) : izan nintzateke aberats 'j'aurais été riche', et p. 42: behar ükhan nükhe 'j'aurais eu besoin', et ce même type apparaît aussi dans le vol. II de la Grammaire de Gavel et Lacombe (1937: 57): «temps $n^{\circ} 22$, conditionnel parfait: ikusi Luke 'il l'aurait vu'; temps $n^{\circ} 23$, conditionnel parfait composé: ikusi ukan Luke 'il l'aurait vu'» (avec auxiliaire redoublé, pour souligner l'antériorité) ${ }^{17}$.

Dans le «Septième tableau préliminaire» de Bonaparte (1869), ce type (son temps composé ${ }^{\circ} 26$ dans cet ouvrage et intitulé «conditionnel présent parfait»), est attribué correctement et au souletin, mais aussi au biscayen, soit repectivement: erori Lizate 'il serait tombé, ikhusi Lüke 'il l'aurait vu', et jausi Lizateke, ikusi L euke. Pour le biscayen encore, voir aussi Campión (1884, p. 598): ikusi L̨eukegu 'él nos lo hubiese visto'. Et, dans le basque (pré) unifié et quelque peu expérimental d'Olabide (1940), on a aussi :

(5a) Yosue'k atsedenera sartu izan balitu, ez L uke Yainkoak beste egun batentzaz arrezkero itz egin. 'Si Josué/Jésus les avait mis au repos, Dieu n’aurait pas parlé après cela d'un autre jour.' (Heb 4,8)

(5b) Bestela, eskeintzaleak, bein garbi eginda gero, ... ez ote Lirake opariok amaitu? 'Autrement, les sacrificateurs une fois purifiés, les offrandes n'auraient-elles pas cessé?' (Heb 10,2)

\subsection{Le type 5: hartu luke(e)n}

Il s'agit de la forme précédente, suffixée du -(e/a)n passé. Il apparaît dans quelques textes, comme la traduction souletine ci-dessous (on reviendra sur diverses autres traductions de ce passage dans la section 4):

(6) Nurk sinhets.i LيüKIen behin ere Abrahamek erranen ahal ziela: Sarak hazten dü ene zahartarzünian egin deitan semia? (Archu, Gen 21,7) lit. 'Qui eût/aurait cru qu'Abraham aurait jamais pu dire: "Sarah nourrit l'enfant qu'elle m’a donné dans ma vieillesse?"'

16. Lallomorphe complexe -te-ke de -ke réservé à izan, 'être' est souvent réduit à -te.

17. Toutes ces formes sont doublées dans ce texte par nos types 1 («temps $n^{\circ} 28$, conditionnel parfait antérieur »: ikusi zukeen, « $\mathrm{n}^{\circ} 29$, conditionnel plus-que-parfait »: ikusi ukan zukeen) et 3 ( $\mathrm{n}^{\circ} 30$, « conditionnel parfait propositif»: ikusiren zukeen »). 
Archu lui-même indique cette forme dans sa Grammaire (1853: 81): agradatü Lizatien ${ }^{18}$ 'il aurait plu' [de 'plaire', avec aux. intransitif], et 'agradatü liatien (avec lia- pour b. commun lira-) au pluriel: 'ils/elles auraient plu'.

Ce type est ausi indiqué, mais seulement pour le biscayen, par Bonaparte (1869: '7 tableau prélim.', n 34), avec jausi Litzatekean / ikusi leukean, même trad. que ci-dessus en 3.1. (Il pourrait s'agir d'un emprunt à Zavala (1848), qui spécifiait que ce tour correspondait à un potentiel très conjectural allant de l'équi-potentiel à l'improbable: ekarri L Łeukean 'podrá ser que él lo hubiese traído' - ce commentaire étant en fait repris par Bonaparte (op.cit., note 5 au même tableau).

\subsection{Le type 6: hartuko lukeen}

Ce dernier type, dans lequel le participe prospectif est maintenant redoublé par le suffixe -ke sur l'aux., est surtout usité dans certaines variétés de biscayen; c'est par ex. le tour qui est systématiquement utilisé dans toute la Bible récente de Kerexeta (1975), à de fausses exceptions près (cf. la section 4. ci-après). Pour ce dialecte, et lui seul, il a été noté par divers auteurs: Zavala (1840:21), Bonaparte (op.cit., $7^{\text {e }}$ tableau prélim. temps $n^{\circ} 35$, « conditionnel passé absolu »), ou encore Dornaku Olaetxea (1910:221).

Il est aussi donné comme alternative au type 3 dans le projet d'unification du biscayen de Villasante et al. (1955), mais aussi dans des projets plus généraux comme Echaide (1944: 100): ura ilko litzakean 'él se habría muerto' intransitif, et (p. 128), ark ilko likekan/ likenan 'él lo habría matado' transitif tutoyé masc/fém., ou encore Ataun (1960: 16), a izango litzaken 'él habría sido'.

Dans les dialectes continentaux, la neutralisation relative (mais voir plus loin) entre l- et $z$ - se trouve chez Darthayet (1902: 99-105), où, par ex. le cond. passé de la conjugaison synthétique du verbe avoir, 'il le lui aurait' est traduit p 104 par zioken, Lioken, zeikon, et avec un objet pl: ziotzaken, L_aizkioken, zeikon; 'il le leur aurait' par zioteken, Lieken; zeiken, et avec l'o.d. pl. ziotzaieken, ㄴieztekin, zeizken (sans spécifications sub-dialectales ni références écrites, malheureusement).

\section{Distinctions sémantico-pragmatiques: note sur les types 2, 3 et 6}

Comme chacun le sait, il y a dans les langues une tension entre l'économie (qui, morphologiquement, mène à certaines ambigüités), et la redondance (qui permet de les lever dans des contextes autrement ambigus). Cette tension peut s'observer dans le cas qui nous concerne ici. Reconsidérons ainsi l'ex. (5), et d'autres trad. de Gen 21,7. Le texte d'Archu luimême en (5) est absolument non-ambigu: dans la première proposition, on a un conditionnel passé en $l$, qui ne saurait s'interpréter autrement - et suggère donc l'idée d'une condition sousentendue comme: 'Si l'on avait demandé à qui que ce soit de prédire le futur...'. Quant à la seconde proposition, ce n'est pas un conditionnel (même enchâssé), mais du discours direct

18. Où le -e de -te se réduit à -i devant voyelle (et parfois ailleurs). 
au présent, qui ne nous concerne pas, à ceci près que toutes les autres traductions auxquelles j'ai eu accès présentent pour cette seconde proposition une FVC de type 3, mais interprétable comme un futur translaté.

Revenant à la FVF de la première proposition, on peut dire que le type 3 (employé par Olabide (1958): Nork esango zioken Abraham'i, Sara'k semea iñutuko ziola?) et la Bible en euskara batua de 1994, ${ }^{19}$ fonctionne comme le type 4 d'Archu, leur FVF étant conditionnelle de manière tout aussi non-ambiguë. Par contre les traductions continentales qui présentent le type 2 (Urte, début du 18e s., Duvoisin 1859-65), restent vagues quant à leur interpétation (vagues, et non ambiguës du point de vue de la question sous-entendue, car il n'y a aucun enjeu particulier). Mais il n'en va pas de même du Type 2 employé par Kerexeta (1976): Nok esango eutsan Abrahan'i, Sara'k semea aziko ebanik? car dans son basque, l'association d'un part. prospectif et d'un aux. passé ne s'interprète que comme un futur translaté. ${ }^{20}$ C'est aussi le cas de la trad. d'Apaolaza (1985): Nork esan bear zion Abrahan-i, Sarak semea aziko zituela bularrean? En effet, ici, l'ambiguité de la première proposition est levée par l'emploi de behar 'devoir', qui régit une forme verbale perfective, et qui surtout pour nous ici, introduit l'idée d'une prédétermination, et est donc incompatible avec la sémantique des conditionnelles.

\section{Une autre valeur pour la combinaison part. prospectif + aux. passé}

A ma connaissance, seul Lafitte (op. cit.: 376, § 708) a mentionné un autre usage de la combinaison qui permet de construire le type 2: «il [=le "futur du passé"] peut marquer une habitude dans le passé», mais il n'en donne qu'un exemple sans source. En fait, ces formes ne sont pas rarissimes, non seulement en navaro-labourdin, mais aussi en guipuzcoan. Faute de place, je n'en donne qu'un pour chaque dialecte: ${ }^{21}$

(6a) Gizon ona zela zioen, sobera edanari emana, zorigaitzez. Emazteak gerla egiten zion, debaldetan: apairu handi batzuetan, bere basoa hutsik utziko zuen bainan Pavlovsky aldean balin bazuen, berdin honen basotik edanen zuen. 'On disait que c'était un homme bon, [mais] malheureusement trop adonné à la boisson. [Sa] femme lui faisait la guerre, en vain: lors de grands repas, parfois, il laissait son verre vide, mais s'il était à côté de Pavlovsky, il buvait même le verre de ce dernier.' P. Larzabal, 1998, Oroitzapenak.

19. Trad. qui ne s'écarte (ici) de la précédente que par le choix lexical du verbe ... et le nombre d'enfants à venir: ... Sarak haurrak izango zituela?

20. la terminaison -(e)nik, pour que (e)la, est surtout usitée quand la principale est négative.

21. J'ai déjà cité pour information un exemple de M. Elissamburu (1890, Frantziako hirur errepubliken ixtoria) et un d'E. Salaberry (1981, Ene sinhestea) dans Rebuschi (2012), mais sans aucune analyse (si ce n'est la suggestion d'une comparaison avec le would dit fréquentatif de l'anglais). J'espère en apporter une brève esquisse ici. [Ajout de dernière minute. En souvenir de lui, qui vient de disparaître, je souhaite aussi signaler que les premières pages (11-13) de l'ouvrage Ene artzain-etxolak, d'Emile Larre, 2001, contiennent 8 exemples d'usage fréquentatif de cette combinaison.] 
(6b) Batzutan, eskatutako [textu] oiek urrengo egunean ekartzen zizkidan. Bestetan, nik eskatu gabea berdin ekarriko zuan.

'Parfois, il m’apportait les textes que je lui avais demandés le jour suivant.

D'autre fois, il en apportait un sans même que je l'aie demandé.'

A. Zabala (1981, vol. I, p 14).

Une fois que l'on accepte l'idée que le suffixe - $k$ o associé au part. perfectif inverse la relation temporelle entre la borne d'un procès la plus proche par rapport à un repère temporel donné (donc la borne qui représente la fin quand le repère suit, et celle qui représente le début quand le repère précède), on comprend que l'on puisse passer d'une perspective temporelle à une modalité, celle d'une forme de prédiction fondée sur l'habitude. (Remarquer à cet égard que la forme en question apparaît dans les textes après un usage non-marqué, avec le participe du verbe lexical à l'imperfectif, aspect grammatical qui exprime généralement l'habitualité.) - On peut même suggérer pourquoi, inversement, l'extension d'usage s'est faite avec -tu-ko, alors qu'a priori, -ke marquant la prédiction aurait pu contribuer à consruire du fréquentatif comme will / would en anglais: c'est sans doute parce que cette valeur sémantique relève précisément de l'aspectualité, bien codée par les trois suffixes -tu, -tu-ko et -tzen, alors que l'usage le plus commun de -ke est associé aux radicaux verbaux qui n'expriment rien de tel.

\section{Dernières remarques}

Bien plus compliquée est la raison pour laquelle le biscayen, et, pour les types 4 et 5 , le souletin aussi, ont opté pour des conditionnels passés (au moins optionnellement) en -l. A cet égard, il faut souligner que les similarités que l'on peut constater entre des deux dialectes (géographiquement périphériques) concernent aussi le le subjonctif passé (construit avec les aux. défectifs), comme le montrent les extraits suivants.

\subsection{Le cas du souletin}

Archu (1853: 92) 'que j'eusse craint: beldür izan nindadin, mais 'qu'il/elle eût craint' beldür izan ledin (pl. : litien). ${ }^{22}$ Gèze (1873), qui admet les deux options. On a ainsi, p. 94, Lekigün / zekigün: 'qu'il - [nous] fût' [ «subjonctif présent ou futur »] en conjugaison synthétique, et: izan Léedin / zédi $n$ : 'qu'il eût été' [« subjonctif plus-que-parfait»]; puis, pour le subj. plusque-parfait, p. 95: 'que j'eusse /qu'il eût conduit' [i.e. 'accompagné'] lagüntü nükiela / lükiela; et encore, p. 109, 'qu'il ait fallu': behar izan Litekeien vs. 'qu'il eût fallu': behar izanzitekeien.

Larrasquet (1934:97) note uniquement, pour le subj. passé: izan néndin / lédin 'que je fusse, qu'il/elle fût'. Mais vu le sujet de ce travail, il faut signaler qu'il fait alterner des conditionnels passés en $z$ - et en l-, tant pour la conjugaison synthétique des verbes forts que

22. Je classe les auteurs chronologiquement. Noter que plus tôt, pour le labourdin, Harriet (1741) proposait aussi: ( p.175) hark atxik Lezan/zezan 'qu'il tînt' et au pl: heiek atxik L_-/z-ezaten 'qu'ils tinssent'; p. 227 hark siñets ㅌezan/zezan 'qu'il crût'. 
pour les formes auxiliaires. Ainsi, p, 95, la conjugaison synthétique d'avoir' est-elle: zükiá ${ }^{23}$ 'il/elle aurait été' (tut.masc.), zükiñá (tut. fém.), zükézün, id. (vouvoiement sg) et enfin zátìn / zátekìn (forme neutre, en s'adressant à plusieurs personnes); avec un sujet absolutif plur., on a: zütükiá 'ils/elles auraient été', zütüküná, zütükézün et ziátìn / ziátekìn respectivement. Mais p. 106-7, toujours en conj. synth., c'est le préfixe l- qui apparaît avec un sujet pluriel: zikedá, 'il/elle m'aurait été' (tut. masc.), mais șitzikedá, au pluriel ('ils/elles m'auraient été), ou encore zikedañá et litzikedañá (id., tut. fém.), zikedázün (id., sg.), mais Litzikedázün 'ils/elles m’auraient été' (vouvoiement); zéikedan 'il/elle m’aurait été (forme neutre) Litzéikedan (pluriel, forme neutre). Aux pp. 107-8, ce sont exactement les même formes qui apparaissent, accompagnant maintenant le participe izan comme verbe principal ou lexical. ${ }^{24}$

\subsection{Le cas du biscayen}

Outre des données équivalentes, il faut remarquer qu'en biscayen (et certaines variétés de guipuzcoan), il y a aussi plus ou moins optionnalité entre l- et z- au potentiel passé. Ainsi, chez Larramendi (op.cit., p. 213-222, 227), on a un l- pour le potentiel; p. 227, il oppose ainsi le (pot.) présent eseri d(a)iteke 'se puede sentar' au "pret[érito] imperf[ecto]" eseri_líteke 'se podía sentar'. (Noter aussi l’absence de $-n$ final, car (ibid.): «el pretérito perfecto [se construye] añadiendo -an.»). De même, Azkue (1924-25, paradigme XLVI), traduit sendatu eukean/Ľeukean par '(él le) podía sanar', la première forme étant "normale et générale » et la seconde, «normale (mais) limitée»; de plus, dans le paradigme XLIX (p. 780) agertu Leitekion est traduit par 'para que pudiera aparecérsele'. Cf aussi eldu Leitekean 'podía o pudo llegar'. A la même époque, Zamarripa (1928: 89-90) donnait un potentiel présent en eldu Leiteke 'puede llegar' et un pot. passé en eldu Leitekean 'podía o pudo llegar', son conditionnel passé étant surcomposé: eldu izango zan 'habría llegado' - mais il employait aussi el type 2, cf. p. 82 : elduko zan 'hubiera llegado et, 'p. 95: artuko eban '(él) hubiera tomado'.

Plus récemment, le collectif Labayru Ikastegia (2002: 314-5) notait aussi que l- (non précédé de ba- 'si', évidemment), associé à une racine d'aux. défectif pouvait se passer du suffixe -ke au potentiel. Or cela est exactement ce que Lafon $(1943,1956)$ avait trouvé dans dans les textes du 16e sècle...

\subsection{En guise de conclusion (temporaire)}

La question est de savoir si ces écarts par rapport à la norme littéraire provient de convergences indépendantes, ou mnifeste au contraire des survivances comme on en trouve

23. Le - $n$ passé disparaît dans les formes allocutives familières. Mais dans certains parlers, elle est plus régulière, et en particulier en biscayen.

24. A un détail près: il semble que la forme de vouvoiement sg. ait été dupliquée pour un sujet pluriel «en parlant à plusieurs personnes » dans la dernière forme proposée : litzikedázün 'ils/ elles m'auraient été'. 
souvent à la périphérie des domaines linguistiques dialectalisés ${ }^{25}$. Si cette seconde option s'avérait soutenable, il faudrait admettre que la très grande régularité morphologique du guipuzcoan (et dans une moindre mesure du labourdin) est en réalité le fruit d'innovations relativement récentes...

\section{Bibliographie}

\section{A. Références grammaticales}

Abréviations sur les sources accessibles via Internet.

$<A R M>$ : textes en $\mathrm{rtf}$ et/ou en pdf, orthographe modernisée, site «Armiarma/Klasikoen gordailua » : http://klasikoak.armiarma.com/alfa.htm./

$<E M D>$ : pdf, «Euskal Memoria Digitala» [Fundación Sancho el Sabio]: http://www. memoriadigitalvasca.es/

$<$ LIKL> : pdf, site «Liburuklik»: http://www.liburuklik.euskadi.net/

$<O A I>$ : pdf, «Gipuzkoako OAI repositorioa » : <http://meta.gipuzkoakultura.net/?locale=es>.

Archu, J.-B. 1853. Grammaire basque-française à l'usage des écoles du Pays basque. Bayonne, Foré $\&$ Lasserre. $<E M D>$.

Ataun, B. 1960. Gure aditza. El verbo vasco. Buenos Aires, Editorial Vasca Ekin.

Aurrekoetxea, G. \& Videgain, X. (éds.). 2004. Haur prodigoaren parabola, Ipar Euskal Herriko 150 bertsiotan. Bilbao, Univ.du Pays basque (Supplément d'ASJU 49).

Aurrekoetxea, G., Videgain, X. \& Iglesias, A. 2007. "Bourciez" bildumako euskal atlas (BBEA), 2: Gramatika. ASJU 39-1.

Azkue, R. 1896. Método práctico para aprender el euskera bizkaino y gipuzkoano. Bilbao, J. de Astuy. $<E M D>$.

—1924-25. Morfología vasca (Gramática básica dialectal del Euskera).. Rééd fac. sim., 3 vol., 1969, Bilbao, La Gran Enciclopedia Vasca.

Bonaparte, L.-L. 1869. Le verbe basque en tableaux. Londres, Strangeways \& Walden. Fac sim. in L-L B., Opera Omnia Vasconice, I (Bilbao : Euskaltzaindia), 175-442. <EMD>.

Campión, A. 1884. Gramática de los cuatro dialectos literarios de la lengua euskara. Tolosa. $<$ LIKL>.

D’Abbadie, A. Th. \& Chaho, J. A. 1836. Études grammaticales sur la langue euskarienne. Paris, Arthus Bertrand.

Darthayet, J.-P. 1861. Guide ou Manuel de la conversation et du style épistolaire français-basque. Bayonne, Lamaignière. $<E M D>$.

— 1867, 1902. Le mécanisme de la construction du verbe basque en dialectes du Labourd et des pays limitrophes. Bayonne, Lamaignière. (Extrait du Guide... 1861). $<E M D>+<L I K L>$.

25. Mais quelles étaient les limites géographiques du basque il y a ne serait-ce que 1.000 ans? 
Dornaku Olaetxea, J. M. R. (1909) Euzkel-iztija ó sea Gramática de la lengua vasca, según el método de Ollendorf. Bilbao, Elexpuru. $<E M D>$.

Echaide, Ignacio. 1944. Desarrollo de las conjugaciones euskaras. San Sebastián, Gráfico Editora. Euskaltzaindia. 2005. Euskal gramatika, lehen urratsak [EGLU] VI-2. Bilbao.

Gavel, H., \& Lacombe, G. 1937. Grammaire basque, II, Le verbe. Bayonne, Courrier.

Gèze, . 1873. Eléments de grammaire basque. Dialecte souletin. Bayonne, Lamaignière. <EMD>.

Harriet, M. 1741. Gramatica escuaraz eta francesez... Bayonne, Fauvet. <EMD>.

Hiriart, A. 1840. Introduction à la langue française et à la langue basque. Bayonne, Cluzeau. $<E M D>$.

Hualde, J. I., Oyharçabal, B. \& Ortiz de Urbina J. 2003. 'Verbs' in Hualde, J.I. \& Ortiz de Urbina, J. (éds), Basque. Berlin-New York, Mouton de Gruyter (195-322).

Inchauspé, E. T. 1858. Le verbe basque. Paris, B. Duprat. $<E M D>$.

Ithurry, A. 1895. Grammaire basque. Dialecte labourdin. Bayonne, Lamaignière. (Rééd. 1920: $<E M D>+<L I K L>)$

Labayru Ikastegia (collectif). 2002. Bizkai Euskeraren Jarraibide Liburua. Bilbao, Labayru Ikastegia.

Lafitte, P. 1944. Grammaire basque (navarro-labourdin littéraire). Bayonne, Librairie «Le Livre». Rééd. facsim., Bayonne, Amis du Musée basque et Ikas. URI/URL de l'éd. de 1944 : <http://hdl.handle.net/10690/468>.

Lafon, R. 1943. Le système du verbe basque au XVle siècle. Bordeaux, thèse en deux vol. Fac.sim., San Sebastián / Donostia, 1980.

_ 1956, Les formes verbales de prétérit à préfixe l- dans les textes du XVI e siècle, rééd. dans R.L., Vasconiana, 1999, Bilbao, Euskaltzaindia, 459-473.

Larramendi, M. 1729. El impossible vencido: Arte de la lengua bascongada. Salamanca, J. Villagordo Alcaraz. Fasim., San Sebastián, Hordago-Lur, 1979. <EMD>.

Larrasquet, J. 1934. Le basque souletin nord-oriental. Paris, Maisonneuve.

Micoleta, R. 1653. Modo breve para aprender la lengua vizcayna. Bilbao. Rééd. 1897, Oxford, James Parker (avec un appendice de E. S. Dodgson). $<E M D>$.

Rebuschi, G. 2012. 'Ultériorité dans le passé et conditionnels en basque du nord'. faits de langues 40, 141-148.

Villasante, L. \& Iturria, C. 1955. Paradigmas de la conjugación vasca (dialectos guipuzcoano y vizcaino). Aranzazu, Editorial Franciscana.

Zamarripa, P. 1928. Gramática vasca. Bermeo, Gaubeka. $<$ EMD>.

Zavala, J. M. 1848. El verbo regular vascongado del dialecto vizcaíno. San Sebastián, Baroja. $<E M D>$.

\section{B. Textes cités ou mentionnés}

\section{B.1. Textes religieuxreligieux}

AnBA [collectif anonyme]. 1828. Jesus-Christo gure Jaunaren Testament Berria. Bayonne, 
Lamaignière. $<$ OAI (sous Leizarraga) $>+{ }^{-}<L I K L>$.

Añibarro, P. A. [ \pm 1800$]$. 1991. Jesu Christoren lau Evangelioac batera alcarturic. Ed. par M. P. Ciarrusta, Bilbao, Jarein. $<A R M>$.

Apaolaza, A. 1985. Asiera. Zamora, Ediciones Monte Casino.

Archü, J.-B. 1862. Moisaren lehen libria, Jenesa deithia. In BOE, Zuberera-1 (1999), 17-112. $<A R M>+<O A I>$.

Archü, J.-B. 1862. Daviden gorantzak edo Psalmiak. Ms. in BOE, Zuberera-1 (1999), 113-242. $<A R M>$.

BOE = Bonaparte ondareko eskuizkribuak. Pagola, M. et al. (éds.). 1992-1999, Bilbao, Deustuko Unibertsitatea (Deiker), 26 volumes.

Bonaparte, L.-L. (†1891). Opera omnia vasconice [=OOV]. [Guvres publiées complètes]. 4 vol. Bilbao, Euskaltzaindia, 1991.

Brunet, F. 1870. Jesu Cristoren evanjelioa Lucasen araura. Londres. Rééd. Madrid, Sociedad Bíblica B. y E., 1909. <EMD>.

Brunet, F. 1883. Jesu Cristoren Evanjelioa Juanen Araura.Londres. <LIKL>.

Casenave-Harigile, J. 1986. Egün oroetako irakurgeiak. Saint-Sébastien, Itxaropena.

—. 2002. Batarzün Berria. Urt/Ahurti (64), Monastère de Beloc.

Cazenave, A. $[ \pm 1860]$. Jesu Khristoin Ebanjelio Seindia San Mathiuin aäbera. Ms., publié in BOE, Ekialdeko Behe-nafarrera-1 (1999), 17-76.

Duvoisin, J.-P. 1859-65. Bible edo Testament Zahar eta Berria [...]. Londres. Facsim.: Bilbao, Gran Enciclopedia Vasca, 1972. <ARM > .

Duvoisin, J.-P. Ms. s.d. Jaun Done Joani Apostoluaren Apokalipza-2. In BOE, Lapurtera-1 (1994), 245-272. <ARM>.

EAB [collectif]. 1994. Elizen Arteko Biblia. Madrid, Sociedades Bíblicas Unidas \& San SebastiánDonostia, E.A.B. Elkartea.

Echenique, B. [ms. \pm 1855 ] 1995. S. Mateoin Evangelioa. In BOE, Iparraldeko Goi-nafarrera-I (1995), 97-176.

Etchehandy, M. 1979. Hasera. Saint-Sébastien, Elkar.

- 1983. Jondoni Pauloren gutunak. Saint-Sébastien, Elkar.

. 1985. Jalgitza; Lebitikoa. Saint-Sébastien, Elkar.

. 1992. Hebrearrei [...] gutunak; Apokalipsia, grekotik euskararat itzuliak. SaintSébastien, Elkar.

2007/2008. Elizen Arteko Biblia [lapurtar-baxenabartarrez]. Urt, Biblia Elkartea \& Belokeko Fraidetxea. < http://amarauna.org/biblia/ >.

Ezkila (collectif anon.) (1974). Jesu Kristoren Berri Ona. [Contient uniquement les 4 Evangiles et les Actes]. Bonloc (Pyrénées Atlantiques), Ed. Ezkila.

Haraneder, J. 1740. Jesu Christoren Evangelio Saindua. Ms., éd. par P. Altuna, Bilbao, Euskaltzaindia, 1990. <ARM>.

Harriet, M. 1855. Iesu-Christo gure Iaunaren Testament Berria. [contient uniquement les quatre 
Evangiles]. Bayonne, E. Lasserre. <LIKL>.

Hualde-Mayo, P. \pm 1850 . Jesu-Kristo gore Jeinaren Ebanjelio Saintiua segun San Mateok, ms. éd. par J. Estornés Lasa, Fontes Lingvee Vasconvm 39 (1982), 43-103. + BOE, Erronkariera-1 (1997), 17-102. <ARM>.

Inchauspé, E. T. 1856. Le saint Evangile selon saint Mathieu, traduit en basque souletin. Bayonne, Veuve Lamaignière. Rééd. OOV-2, 91-150. $<$ ARM $>+<O A I>$.

Inchauspé, E. T. 1858. L'Apocalypse de l'apôtre Saint Jean, traduite en basque souletin. Londres 1858. Rééd. in OOV-1, 275-307. <ARM > + <OAI>.

Iribarnegaray, X. [ $[$ 1860]. Yesu Kristoin Ebanjeliua San Mathioin arabera. In BOE, Mendebaldeko behe-nafarrera (1999), p.17-81.

Kerexeta, J. 1976. Euskal-Biblia (bizkaieraz). Bilbao, Bilboko Elizbarrutiko Gotzaintza.

Léon, L. 1946. Jesu-Kristo gure Jaunaren Ebanjelio Saindua. Ustaritz, publié par l'auteur.

Liçarrague / Leizarraga, J. 1571. Iesus Christ Gure Iaunaren Testamentu Berria [etc.] La Rochelle. Facsim. par Th. Linschmann \& H. Schuchardt, Strasbourg, $1900<$ OAI>. Facsim. de ce dernier, Bilbao, Euskaltzaindia, 1990. <ARM>.

Lizarraga, J. [ \pm 1820$]$ (1868). Jesucristoren Evangelio Saindua, Juanec dacarran guisara. Londres. Fac-sim., oov III, 13-102. ms. ed. in BOE, Hegoaldeko Goi-nafarrera-4 (1996), 12351275. <ARM> [sous: Beste zenbait itzulpen]

Olabide, R./E.1931. Itun berria. $<E M D>$.

—. 1958. Idazteuna. Itun Zaar eta Berria. Bilbao, Yesuren Biotzaren Deya. IInclut l'entrée précédente].

OOV. Voir Bonaparte.

Orixe [Ormaetxea, N., dit -]. 1974. Jesu Krito gure Jaunaren Berri Ona. In Orixe, Kerexeta \& Zugasti, Itun berria², San Sebastián, Lazkano'ko Beneditarrak, 5-237.

Otaeg(u)i, C. [ 1860]. San Mateoren Ebanjelioa Ceamaco escuera itzulia. In BOE, Gipuzkera-5 (1993), 2799-2852.In BOE, Gipuzkera-5 (1993), 2799-2852.

Oteiza ("Médico"). 1838. Evangelioa, San Lucasen Guissan. Madrid, Imprenta de la Compañia Tipográfica. $<E M D>$.

Salaberry, E. 1978. Ene sinestea. Saint-Sébastien, Zabal. Rééd. Susa: <http://www.susa-literatura.com/emailuak/salaberri/>.

Salaberry, M., dit d'Ibarrole. 1856. L'Evangile selon Sant-Matthieu [...]. Bayonne.

Bayonne, Veuve Lamaignère. Facsim., OOV III, 335-420. <OAI>.

Samper, P. J. 1869. Jesucristo gure Jaunaren Evangelio sandua segun San Mateo. Ms., in BOE, Zaraitzera (1997), 17-81.

Udabe, J. E. [ \pm 1857$]$. Evangelio santu gure Jesu Cristo Jaunarena, Done Matheoren arauran... Ms., In BOE, Gipuzkera-4 (1993), 1969-2024. — Version corrigée par L.-L. B. de 1,1 à 8,28, Londres (1857) : facsim. in OOV, IV, 11-29.

Uriarte, J. A. [ \pm 1860$]$. Biblia. Ms, éd. in BOE, gipuzkera-1-3+ 4 (1969-2305). <ARM>.

— [ \pm 1860$]$. San Mateoren Ebanjelioa [en biscayen]. Ms, éd. in BOE, bizkaiera-1, 183-254.

Ürrüthy, A. 1873a. Ebanjelio Saintia Jesus-Kristena Jondane Johaneren arabera. Bayonne, 
Imprimerie Casals. <LIKL>

Ürrüthy, A., 1873b, Jondane Phetiriren Epitriac. Bayonne, Imprimerie Casals. <OAI>

d’Urte, Pierre. \pm 1710 . Moisseren lehenbicico libúruä Ieneraçionea edo Etórqiä deitúä. London: Trinitarian Bible Society, 1898. $<A R M>+<E M D>$.

\section{B.2. Autres textes}

Larre, E. 2001. Ene artzain-etxolak. Oyarzun, Sendoa (Auspoan Liburutegia 273).

Larzabal, P (1915-1988). 'Hazparne' In P. Charritton (éd.), Piarres Larzabalen idazlanak. VII, Oroitzapenak. Saint-Sébastien, Elkar. $<A R M>$.

Zavala, A. 1981. 'Itzaurrea'. In I. Alkain \& A. Zavala, Gerrateko ibillerak, I. Tolosa, Editorial Auspoa, Auspoa liburutegia 148 (9-23). 